\title{
BMJ Open Is the Yale Global Tic Severity Scale a valid tool for parent-reported assessment in the paediatric population? A prospective observational study in Taiwan
}

\author{
Che-Sheng Ho, ${ }^{1,2}$ Jia-Yun Huang, ${ }^{2}$ Chien-Hui Yang, ${ }^{2}$ Yi-Jie Lin, ${ }^{1,2}$ \\ Ming-Yuan Huang (D) , ${ }^{1,3}$ Yung-Cheng Su (D) 4,5
}

To cite: Ho C-S, Huang J-Y, Yang $\mathrm{C}-\mathrm{H}$, et al. Is the Yale Global Tic Severity Scale a valid tool for parentreported assessment in the paediatric population? A prospective observational study in Taiwan. BMJ Open 2020;10:e034634. doi:10.1136/ bmjopen-2019-034634

- Prepublication history for this paper is available online. To view these files, please visit the journal online (http://dx.doi. org/10.1136/bmjopen-2019034634).

$\mathrm{M}-\mathrm{YH}$ and $\mathrm{Y}-\mathrm{CS}$ contributed equally.

Received 15 0ctober 2019 Revised 04 May 2020 Accepted 19 June 2020

Check for updates

(C) Author(s) (or their employer(s)) 2020. Re-use permitted under CC BY-NC. No commercial re-use. See rights and permissions. Published by BMJ.

For numbered affiliations see end of article.

Correspondence to Dr Yung-Cheng Su; drsu119@gmail.com

\section{ABSTRACT}

Objective The Yale Global Tic Severity Scale (YGTSS) is the most commonly used clinician-rated evaluation tool for Tourette syndrome (TS), with established reliability and validity. This study aims to determine whether the YGTSS is a valid parent-reported assessment in the TS population.

Design A prospective cohort study.

Setting A major medical centre in Taiwan.

Methods A total of 594 patients were enrolled. A revised traditional Chinese version of the YGTSS was made available to parents via Google docs. Parents were encouraged to complete the YGTSS the day before each outpatient clinic visit. At each visit, a paediatric neurology fellow also administered the YGTSS assessment. We investigated whether differences in scores between physicians and parents changed as the number of parent evaluations increased. The results of the physician assessments were also taken as the expert standard for evaluating the sensitivity and specificity of the parentreported assessments was conducted for the same visit. Results The differences in the YGTSS scores between participants and physicians were small. The mean difference in the total assessment score was 4.15 points. As the number of times the parent evaluation was performed increased, the difference between the parent and physician scores decreased. Discrimination of moderate-to-severe attacks was good using the parent-assessed YGTSS (area under the receiver operating characteristic curve, $0.858 ; 95 \% \mathrm{Cl} 0.839$ to 0.876$)$. The sensitivity for detecting a moderate-to-severe attack by YGTSS parent assessment was 79.7\% (95\% Cl 76.6 to 82.8$)$, and the specificity was $91.8 \%$ (95\% $\mathrm{Cl} 89.9$ to 93.7$)$. Conclusion The parent-reported YGTSS is a promising tool for TS assessment, demonstrating good discriminative ability for disease severity, with user precision increasing with experience.

\section{INTRODUCTION}

Tourette syndrome (TS) is characterised by persistent motor and vocal tics that begin before 18 years of age, and it is estimated to affect 6 per 1000 children. ${ }^{1}$ The clinical presentation of TS is complex, as the symptoms may wax and wane in frequency, intensity and type. ${ }^{23}$ The severity
Strengths and limitations of this study

- This study evaluated the hypothesis that the Yale Global Tic Severity Scale (YGTSS) is a valid tool for parent-reporting, allowing for better communication and decision-making between doctors and patients.

- It is difficult to train many parents repeatedly to ensure them to achieve an acceptable level before they posted their scores, and the internal reliability may be difficult to be evaluated.

- There may also have been variability in the YGTSS evaluations from pedestrians.

is influenced by multiple factors, including stress and social interactions, ${ }^{4-6}$ making clinical assessment challenging. The most widely used measure to assess the severity of TS is the Yale Global Tic Severity Scale (YGTSS), ${ }^{78}$ a clinicianadministered, semistructured interview that assesses tic and tic-related impairment severity over the previous week.

The YGTSS includes a symptom checklist for motor and vocal tics. Both motor and vocal tics are assessed for symptom number, frequency, intensity, complexity and interference on a 0-5 Likert scale. Scores from each dimension are totalled to reflect the severity of motor tics (range 0-25), vocal tics (range 0-25) and combined tics (range 0-50). A separate ticrelated impairment scale, scored from 0 to 50 , is also included. Although several other assessments have been developed, the YGTSS is still the most commonly used, with established reliability and validity. ${ }^{79-12}$

In practice, clinicians do rely in part on patient report to make their assessment; that is, not all tics present during the interview. ${ }^{13}$ The use of patient-reported outcome measures (PROMs) has the potential to narrow the gap in clinical manifestations observed between clinicians 
and patients and to help adjust treatment plans. ${ }^{14}{ }^{15}$ Several self-report instruments for TS have been developed for this purpose. The Proxy Report Questionnaire for Parents and Teachers and the Apter 4-questions are limited by insufficient validation and relatively low specificity. ${ }^{12} 1617$ The Premonitory Urges for Tics Scale has shown good psychometric properties; however, it is not acceptable for patients younger than 10 years of age. ${ }^{12} 18$ This study evaluates the hypothesis that YGTSS is a valid tool for parent-reporting in the TS population. Such a tool would allow for better communication and decision-making between doctors and patients, and patient satisfaction regarding their care may also improve.

\section{METHODS}

\section{Participants}

Data collection

This study was approved by the Institutional Review Board of Taipei Mackay Memorial Hospital, Taiwan. A database was created to collect patient information. Paediatric patients with TS who are regularly followed up in the Taipei Mackay
Memorial Hospital were enrolled after informed consent was provided by their parents. The authors carried out a Chinese translation of the YGTSS. Physicians in the Division of Paediatric Neurology, Department of Paediatrics, MacKay Children's Hospital, Taipei, Taiwan reviewed the contents to reach a consensus, and differing perspectives were resolved by group discussions. Beginning in June 2018, a revised traditional Chinese version of the YGTSS was made available to parents via Google docs (figure 1). On introduction of the assessment to parents, a paediatric neurologist explained the use of the assessment scales to make sure parents clearly understood how to rate their symptoms. Parents were encouraged to complete the YGTSS the day before each outpatient clinic visit. On the date of the visit, a paediatric neurology fellow was assigned to the patients by convenience sampling in the waiting room and also administered the YGTSS. The parents and the paediatric fellows were blind to the YGTSS results of the other. Some patients were administered the YGTSS evaluation by both the parents and the paediatric fellow during the same visit. The attending physicians used

\begin{tabular}{|c|c|}
\hline 過去一週内绝的賽貝發生「動作型」症狀的湩頼 & Figure1.motor tic symptom during the past week \\
\hline 簡單型 & Simple \\
\hline 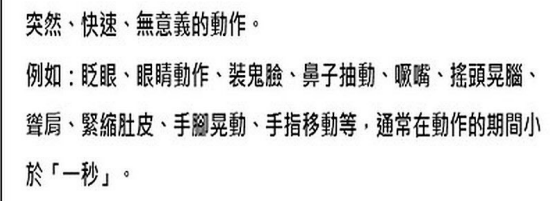 & $\begin{array}{l}\text { A sudden, brief, "meaningless" movement that recursin bouts } \\
\text { such as eye blinking, eye rolling, grimacing, nose-twitching, head } \\
\text { jerking, shoulder shrugging, abdominal tensing, limb jerking and } \\
\text { finger moving. The attacks are often last than } 1 \text { second. }\end{array}$ \\
\hline Complex & Complex \\
\hline 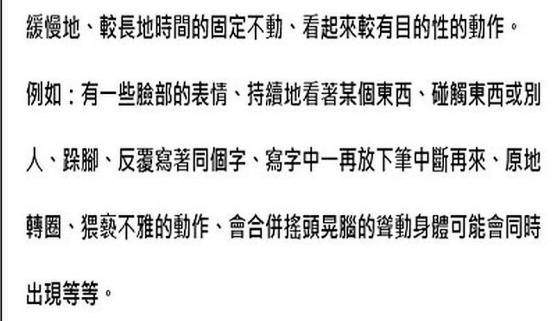 & $\begin{array}{l}\text { Stereotyped semi-purposeful movements with longer duration } \\
\text { Such as facial grimacing together with body movements, looking to } \\
\text { one side for a brief period of time, touching objects and other } \\
\text { people, stomping or tapping the foot, writing over and over the } \\
\text { same letter or word, pulling back on the pencil while writing, } \\
\text { twirling, Obscene movements or gestures. }\end{array}$ \\
\hline 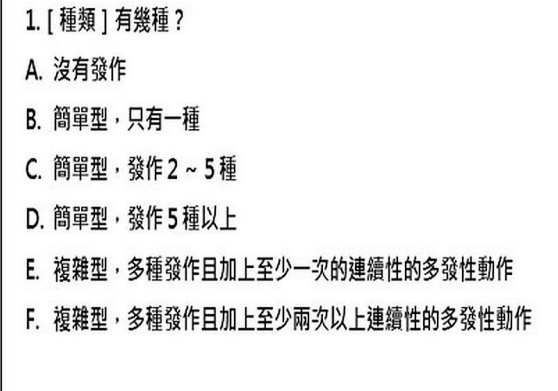 & $\begin{array}{l}\text { 1. How many kinds of symptoms have you noticed? } \\
\text { A. None } \\
\text { B. Single tic } \\
\text { C. Multiple discrete tics (2-5) } \\
\text { D. Multiple discrete tics plus as least one orchestrated pattern of } \\
\text { multiple simultaneous or sequential tics } \\
\text { E. Multiple discrete tics plus several (>2) orchestrated paroxysms of } \\
\text { multiple simultaneous or sequential tics }\end{array}$ \\
\hline
\end{tabular}

Figure 1 Revised traditional Chinese version of the Yale Global Tic Severity Scale made available via Google docs. 
the YGTSS results as a reference for making medical decisions during the visit. Patient age and sex, date of visit and parent-assessed or paediatric-fellow-administered YGTSS scores were recorded.

\section{Statistical analyses}

We first evaluated the absolute differences in the YGTSS scores by subtracting the scores of parents from that of physicians. We also assessed the difference between the two measurements across multiple visits using linear regression. To adjust for correlations in the data due to being collected at multiple times by the same participants, the generalised estimating equation (GEE) method $^{19}$ was adapted to account for clustering of participants in the evaluation of score differences.

We also dichotomised tic attack as mild or moderate/ severe by defining a mild attack as a YGTSS score $<20$ and a moderate-to-severe tic attack as $>20 .^{20}$ The discriminatory power of the parent-reported YGTSS for a moderate-tosevere attack was assessed by using the area under the receiver operating characteristic curve (AUROC) based on a logistic regression model with GEE. Feedback from the parents was collected by convenience sampling at outpatient clinics. All $p$ values were two-tailed, and $\mathrm{p}<0.05$ was considered statistically significant. All analyses were performed using Statistical Analysis Software for Windows, V.9.4 (SAS Institute, Cary, NC, USA).

\section{Patient and public involvement}

Patients and the public were not involved in the design or planning of this study.

\section{RESULTS}

\section{Study population}

A total of 594 patients were enrolled in this study between June 2018 and April 2019, with 3356 evaluations contributed by their parents. On average, each participant contributed 5.65 parent-reported YGTSS evaluations during the study period. Among these parent reports, 1455 were paired with simultaneous evaluations by paediatric fellows and were used for analyses. The final analysis included 527 patients. The mean patient age was 8.8 years (SD, 2.97), and $82.5 \%$ $(n=435)$ of the patients were men. A flow chart of the patient selection process is illustrated in figure 2.

\section{Comparison of assessment scores between participants and physicians}

The differences in the YGTSS scores between participants and physicians were small (table 1). The mean difference in the total assessment score was 4.15 points, with the greatest difference being for 'tic-related impairments'. As the number of times the parent evaluation was completed increased, the difference between the parent and physician scores decreased. After taking parent clustering into account, the absolute difference in total scores between participants and physicians decreased by 0.24 points $(95 \%$ CI 0.14 to $0.34 ; \mathrm{p}<0.001$ ) for each repetition of the assessment.

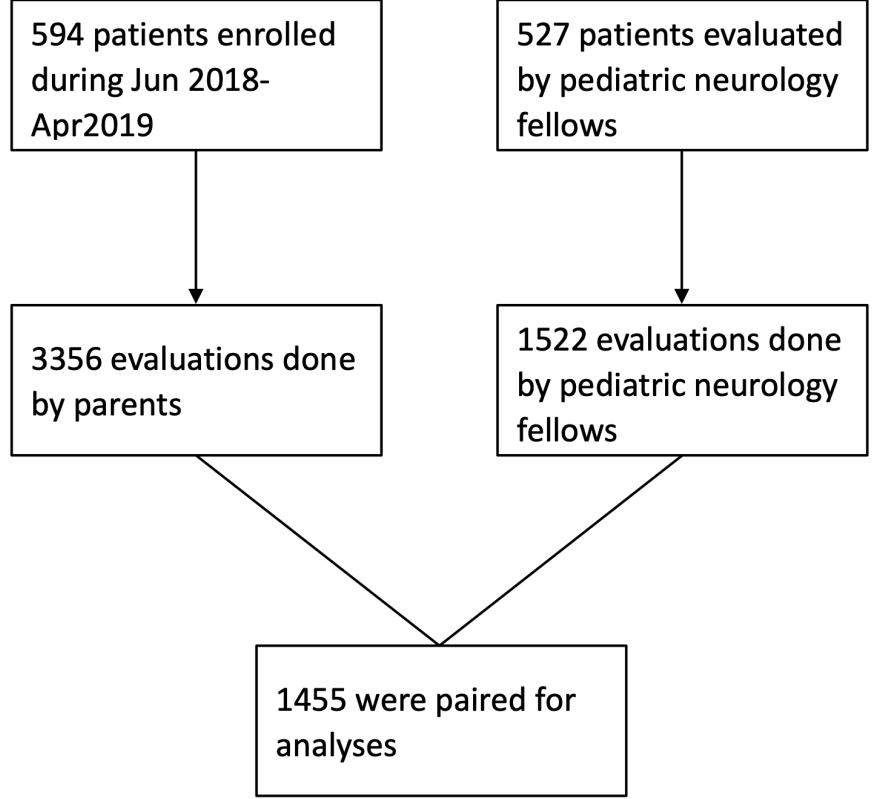

Figure 2 Flow chart of patient selection.

A subgroup analysis of the combined tic severity category revealed an absolute average difference of 2.40 points. The absolute difference in combined tic severity decreased by 0.17 (95\% CI 0.11 to $0.22 ; \mathrm{p}<0.001)$ for each repetition of the assessment. After participants completed the assessment four times, the difference between participant and physician scores was no longer significant (figure 3).

\section{Diagnostic accuracy of the YGTSS parent evaluation}

The power of discriminating moderate-to-severe attacks with the YGTSS parent assessment was good (AUROC, $0.858 ; 95 \%$ CI 0.839 to 0.876 ). The specificity for detecting a moderate-to-severe attack using the YGTSS parent assessment was significantly high. Of 819 physician assessments of mild attacks, 752 were in accordance with that of the parents, yielding a specificity of $91.8 \%$ (95\% CI 89.9 to 93.7 ). In 636 physician assessments of moderate-to-severe attacks, 507 were in accordance with that of the parents, yielding a sensitivity of $79.7 \%$ (95\% CI 76.6 to 82.8 ).

Table 1 Comparison of parent-assessed and physicianassessed YGTSS scores according to assessment category*

\begin{tabular}{lll}
\hline Assessment category & $\begin{array}{l}\text { Mean } \\
\text { difference } \\
\text { (points) }\end{array}$ & $\mathbf{9 5 \%} \mathbf{~ C l}$ \\
\hline $\begin{array}{l}\text { Entire assessment (all } \\
\text { categories) }\end{array}$ & 4.15 & 3.82 to 4.48 \\
\hline Motor tic severity & 1.17 & 1.07 to 1.28 \\
\hline Vocal tic severity & 1.23 & 1.11 to 1.35 \\
\hline Combined tic severity & 2.40 & 2.22 to 2.58 \\
\hline Tic-related impairment & 2.41 & 2.14 to 2.68 \\
\hline
\end{tabular}

${ }^{*} \mathrm{n}=1455$.

YGTSS, Yale Global Tic Severity Scale. 


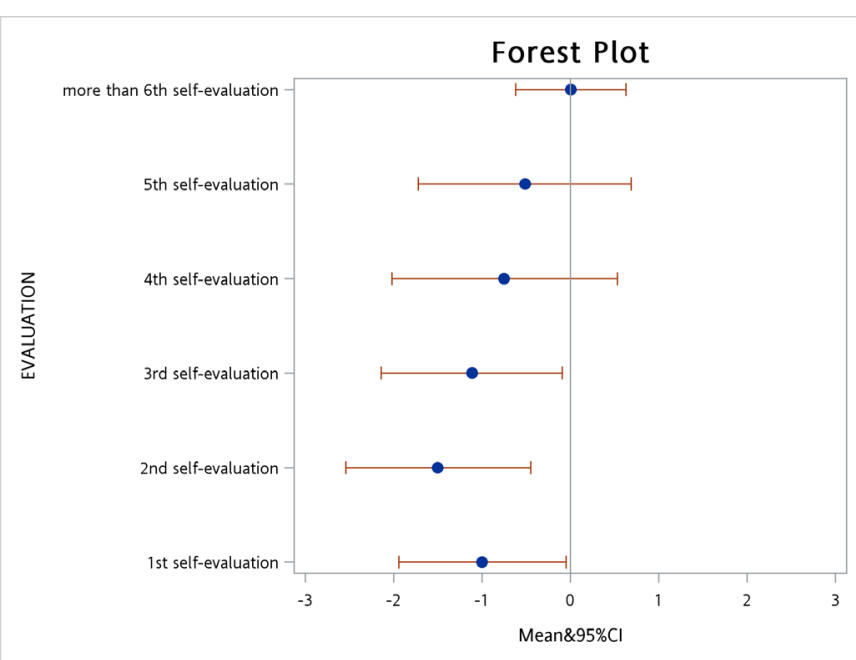

Figure 3 Distribution of average score differences.

\section{Evaluation of feedback}

Most comments from participants were positive, as the following examples indicate:

1. After assessment of my child, I know better what the doctor needs to know, and this process also helps me better understand how to take care of my child.

2. With these long-term, objective trends in my results, I think discussing the goals of treatment with doctors is clearer.

Feedback taken by convenience sampling from physicians at hospital outpatient clinics was also encouraging:

1. Being able to understand the patient's condition outside of the hospital allows me to communicate more effectively with caregivers.

\section{DISCUSSIONS}

The aim of the present study was to evaluate the potential use of the YGTSS as a parent-reported measure of tic severity in children with TS. The results showed an overall good ability to discriminate a moderate-to-severe TS attack via parentreporting (AUROC, 0.858). The sensitivity and specificity for detecting a moderate-to-severe TS attack were reasonably high. With repeated practice responding to the assessment, the parent-reported scores became similar to those of physicians, with no difference after the fourth assessment. Our results indicate that the YGTSS, the most widely used TS assessment tool, may be as accurate when used by a child's parent as it is when administered by the child's clinician.

In this study, we used a step-by-step online Google doc interface to help the participants fill out the forms with little difficulty. The online parent-reported YGTSS database also allowed participants to complete the evaluation without time and space limits, and more than 3000 parent evaluations submitted during the study period are one factor contributing to the efficiency of the system. The feedback from both parents and clinicians was positive, and the database continues to grow as the number of parent-reported submissions increases. Paediatric neurologists currently often rely on parent-reported assessments to adjusting treatment plans. ${ }^{34}$ As self-assessments allow parents and clinicians to share the same information regarding a patient's condition, the communication is more fluent and efficient. ${ }^{21} 22$

Another reason for our positive results is that the parents were aware of the disease and highly motivated to be involved in the management of their child's TS. They may be more likely to present precise evaluations if possible. During the multiple interactions about the conditions with their clinicians, parents became more practiced and accurate with their evaluations. Patients generally welcome systems that routinely use PROMs. ${ }^{14}$ The parent-reported YGTSS correlates highly with factors that have value to clinicians. Even for the clinician-administered YGTSS, the interviewer relies heavily on patients' and their family members' insights, as patients may not present with the full range of tics during the interview. As a result, parent-reporting may more closely reflect the actual patient condition.

\section{Limitations}

Our study has several limitations. First, the participants were parents of children with TS, and most of them had already participated in regular follow-up at our outpatient clinics. Thus, these parents may have been more aware of their child's symptoms, allowing for an easier understanding of the YGTSS parameters, resulting in a high correlation between the responses of the parents and physicians. Second, as more than 500 patients were included in the database, it was difficult to provide parents intensive training to ensure that they had achieved an acceptable level of performance before they began submitting their scores; thus, the internal reliability may be difficult to be evaluated. There may also have been variability in the YGTSS evaluations from paediatric fellows. However, these results are representative of real clinical situations. Third, the paediatric fellows visit and evaluate the patients in the waiting room by convenience sampling, which may have led to sampling bias. Fourth, in our cohort there were only a few patients newly diagnosed with TS. As a result, we were unable to perform subgroup analyses for these patients, comparing between those whose child was recently diagnosed and thus were less familiar with the symptoms versus those whose child had the diagnosis for quite a while and were therefore very familiar with the symptoms. We also did not adjust for important patient characteristics such as severity of tics and duration since initial diagnosis as that information was lacking. Lastly, the evaluations from the physicians were not performed simultaneously with the participants. Since the physicians evaluated information by directly observing patients, the symptoms may have differed from those at the time of the parent-reporting.

\section{CONCLUSION}

The parent-reported YGTSS is a promising tool for TS assessment, demonstrating good discriminative ability for disease severity, with user precision increasing with experience.

Author affiliations

${ }^{1}$ Department of Medicine, MacKay Medical College, New Taipei City, Taiwan 
${ }^{2}$ Division of Pediatric Neurology, Department of Pediatrics, MacKay Children's Hospital, Taipei, Taiwan

${ }^{3}$ Department of Emergency Medicine, Mackay Memorial Hospital, Taipei, Taiwan ${ }^{4}$ Emergency Department, Dalin Tzu Chi Hospital, Buddhist Tzu Chi Medical Foundation, Chiayi, Taiwan

${ }^{5}$ School of Medicine, Tzu Chi University, Hualien, Taiwan

Acknowledgements The authors thank Dr Chen-Yang Hsu (Institute of Epidemiology and Preventive Medicine, College of Public Health, National Taiwan University, Taipei, Taiwan) for the in-depth discussion on statistical methods. The authors thank Dr James F Leckman (Yale Child Study Center, New Haven, Connecticut, USA) and Dr Jung-Chieh Du (Department of Pediatrics, Taipei City Hospital, Zhongxiao Branch, Taipei, Taiwan) for the development of Chinese version of Yale Global Tic Severity Scale.

Contributors M-YH analysed and interpreted the data. J-YH, C-HY and Y-JL interpreted the data and contributed to the manuscript development. C-SH supervised the study and interpreted the data. Y-CS analysed the data, and was a major contributor in writing the manuscript. All authors read and approved the final manuscript.

Funding The authors have not declared a specific grant for this research from any funding agency in the public, commercial or not-for-profit sectors.

Competing interests None declared.

Patient consent for publication Not required.

Ethics approval This study was initiated after approval from the Institutional Review Board of Taipei Mackay Memorial Hospital, Taiwan.

Provenance and peer review Not commissioned; externally peer reviewed.

Data availability statement № data are available.

Open access This is an open access article distributed in accordance with the Creative Commons Attribution Non Commercial (CC BY-NC 4.0) license, which permits others to distribute, remix, adapt, build upon this work non-commercially, and license their derivative works on different terms, provided the original work is properly cited, appropriate credit is given, any changes made indicated, and the use is non-commercial. See: http://creativecommons.org/licenses/by-nc/4.0/.

ORCID iDs

Ming-Yuan Huang http://orcid.org/0000-0002-1501-9893

Yung-Cheng Su http://orcid.org/0000-0002-8201-1592

\section{REFERENCES}

1 Jeon S, Walkup JT, Woods DW, et al. Detecting a clinically meaningful change in tic severity in Tourette syndrome: a comparison of three methods. Contemp Clin Trials 2013;36:414-20.

2 Abramovitch A, Reese H, Woods DW, et al. Psychometric properties of a self-report instrument for the assessment of tic severity in adults with tic disorders. Behav Ther 2015;46:786-96.

3 Lin H, Yeh C-B, Peterson BS, et al. Assessment of symptom exacerbations in a longitudinal study of children with Tourette's syndrome or obsessive-compulsive disorder. J Am Acad Child Adolesc Psychiatry 2002;41:1070-7.

4 Eapen V, Fox-Hiley P, Banerjee S, et al. Clinical features and associated psychopathology in a Tourette syndrome cohort. Acta Neurol Scand 2004;109:255-60.

5 Chang S, Himle MB, Tucker BTP, et al. Initial psychometric properties of a brief Parent-Report instrument for assessing tic severity in children with chronic tic disorders. Child Fam Behav Ther 2009;31:181-91.

6 Silva RR, Munoz DM, Barickman J, et al. Environmental factors and related fluctuation of symptoms in children and adolescents with Tourette's disorder. J Child Psychol Psychiatry 1995;36:305-12.

7 Leckman JF, Riddle MA, Hardin MT, et al. The Yale global tic severity scale: initial testing of a clinician-rated scale of tic severity. $J \mathrm{Am}$ Acad Child Adolesc Psychiatry 1989;28:566-73.

8 Ho C-S, Chiu N-C, Tseng C-F, et al. Clinical effectiveness of aripiprazole in short-term treatment of tic disorder in children and adolescents: a naturalistic study. Pediatr Neonatol 2014;55:48-52.

9 Storch EA, Murphy TK, Geffken GR, et al. Reliability and validity of the Yale global tic severity scale. Psychol Assess 2005;17:486-91.

10 Storch EA, De Nadai AS, Lewin AB, et al. Defining treatment response in pediatric tic disorders: a signal detection analysis of the Yale global tic severity scale. J Child Adolesc Psychopharmacol 2011;21:621-7

11 Ho C-S, Chen H-J, Chiu N-C, et al. Short-Term sulpiride treatment of children and adolescents with Tourette syndrome or chronic tic disorder. J Formos Med Assoc 2009;108:788-93.

12 Martino D, Pringsheim TM, Cavanna AE, et al. Systematic review of severity scales and screening instruments for tics: critique and recommendations. Mov Disord 2017;32:467-73.

13 Conelea CA, Woods DW. The influence of contextual factors on tic expression in Tourette's syndrome: a review. J Psychosom Res 2008;65:487-96.

14 Nelson EC, Eftimovska E, Lind C, et al. Patient reported outcome measures in practice. $B M J$ 2015;350:g7818.

15 Barry MJ, Edgman-Levitan S. Shared decision making--pinnacle of patient-centered care. N Engl J Med 2012;366:780-1.

16 Cubo E, Sáez Velasco S, Delgado Benito V, et al. Validation of screening instruments for neuroepidemiological surveys of tic disorders. Mov Disord 2011;26:520-6.

17 Linazasoro G, Van Blercom N, de Zárate CO. Prevalence of tic disorder in two schools in the Basque country: results and methodological caveats. Mov Disord 2006;21:2106-9.

18 Woods DW, Piacentini J, Himle MB, et al. Premonitory urge for tics scale (puts): initial psychometric results and examination of the premonitory urge phenomenon in youths with tic disorders. J Dev Behav Pediatr 2005;26:397-403.

19 Zeger SL, Liang KY. Longitudinal data analysis for discrete and continuous outcomes. Biometrics 1986;42:121-30.

20 Sallee F, Kohegyi E, Zhao J, et al. Randomized, double-blind, placebo-controlled trial demonstrates the efficacy and safety of oral aripiprazole for the treatment of Tourette's disorder in children and adolescents. J Child Adolesc Psychopharmacol 2017;27:771-81.

21 Crandall W, Kappelman MD, Colletti RB, et al. ImproveCareNow: the development of a pediatric inflammatory bowel disease improvement network. Inflamm Bowel Dis 2011;17:450-7.

22 Lannon CM, Peterson LE. Pediatric collaborative networks for quality improvement and research. Acad Pediatr 2013;13:S69-74. 